\title{
Approximated expressions for the coherent synchrotron radiation effect in various accelerator scenarios
}

\author{
Donish Z. Khan ${ }^{*}$ and Tor O. Raubenheimer $\oplus^{\dagger}$ \\ SLAC National Accelerator Laboratory, Menlo Park, California 94025, USA
}

(Received 23 April 2021; accepted 30 July 2021; published 20 August 2021)

\begin{abstract}
In this paper, we present analytic expressions for the coherent synchrotron radiation (CSR)-induced rms energy spread and the resulting emittance dilution due to three common scenarios in electron particle accelerators: An electron bunch transitioning into the steady-state regime while traversing a bending magnet with and without the effects of compression, and an electron bunch coasting into a subsequent drift section. The expressions are used to calculate the CSR-induced rms energy spread and emittance dilution for real bunch compressor systems: the first and second bunch compressors of the LCLS-II CuRF and LCLS-II-HE. The expressions are compared with simulations using ELEGANT for various parameter scans.
\end{abstract}

DOI: $10.1103 /$ PhysRevAccelBeams.24.080701

\section{INTRODUCTION}

In today's $\mathrm{x}$-ray free electron laser (XFEL), the FEL radiation's dependence on emittance has motivated research to understand the various emittance dilution mechanisms to ensure the maximal output of the laser. One such mechanism is the coherent synchrotron radiation (CSR) effect. The effect can be prominent in parts of an FEL where the bunch length is short, the peak current is high and the synchrotron radiation emitted in the bending magnets is coherent. Though the CSR effect has been comprehensively studied, it has yet to be quantified for a variety of accelerator scenarios-for example, bunch compressors, quadrupole magnets, and undulator transients [1-9]. In fact, the CSR rms energy spread has only been calculated for a Gaussian beam traversing a dipole or undulator magnet in the steady-state regime $[10,11]$. The current CSR expressions are limited in application and computational mobility as they can only be solved numerically $[10,12,13]$. There is a practicality in establishing expressions that are accessible for order-of-magnitude estimates and to provide perspective on the parameter scaling.

In this paper, we describe the development of analytic expressions for the CSR-induced rms energy spread resulting from three typical scenarios for electron particle accelerators to which there are currently no known

\footnotetext{
*donish@slac.stanford.edu

tor@slac.stanford.edu
}

Published by the American Physical Society under the terms of the Creative Commons Attribution 4.0 International license. Further distribution of this work must maintain attribution to the author(s) and the published article's title, journal citation, and DOI. solutions. Although three-dimensional effects can be important for most applications of current interest, the one-dimensional (1D) theories are sufficient [14-17] and we limit ourselves to the 1D models.

The paper is organized as follows. In Sec. II, we give an overview of the 1D CSR model providing the foundation of our formalism. Section III outlines the derivation of the three CSR formulas followed by extensive parameter scans with simulations. Finally, in Sec. IV, we apply our formulas to the bunch compressor systems (BC1 and BC2) of the LCLS-II $\mathrm{CuRF}$ and LCLS-II-HE to calculate the energy spread and projected emittance growth due to CSR and compare the results with that of simulations [18,19]. All simulation results in this paper have been obtained using ELEGANT [20].

\section{1D CSR THEORY}

The standard derivation of the CSR wakefield begins with the Liénard Wiechert fields for two electrons traveling along the same trajectory, ignoring any transverse deviation from the on-energy path, the so-called 1D projected model [12]. The energy transfer due to the electric field of the trailing electron onto the observing electron is given by $\frac{d E}{d(c t)}=e \vec{\beta} \cdot \vec{E}$. The wakefield is inversely proportional to the distance between the two electrons and contains a singularity when the electron separation approaches zero. The singularity was removed by the normalization process developed by Saldin et al., by subtracting off the contribution of two electrons traveling along a straight line [12]. The resulting two-electron CSR kernel can then be integrated over the retarded longitudinal bunch distribution (assuming the variation of the longitudinal distribution is much smaller than the characteristic wavelength of coherent radiation) to give the collective effect under a wide range of scenarios one might find in an accelerator 
environment. In particular, we focus on two cases: an electron bunch entering a bending magnet and transitioning towards the steady-state regime which arises when the radiation from the entire tail of the electron bunch overtakes the head in a uniform bend, and an electron bunch exiting a bend and coasting into the subsequent drift section. Both are examined in the ultrarelativistic regime $(\gamma \rightarrow \infty)$.

The complete mathematical expressions for these cases under the 1D projected model are as follows [13]

$$
\begin{aligned}
\left.\frac{d E}{d s}\right|_{\text {Bend }}= & \frac{4 e^{2}}{\rho \phi}\left(\lambda\left(z-z_{B}\right)-\lambda\left(z-z_{A}\right)\right) \\
& +\frac{2 e^{2}}{\left(3 \rho^{2}\right)^{1 / 3}} \int_{z-z_{B}}^{z} \frac{1}{\left(z-z^{\prime}\right)^{1 / 3}} \frac{\partial \lambda\left(z^{\prime}\right)}{\partial z^{\prime}} d z^{\prime}, \\
\left.\frac{d E}{d s}\right|_{\text {Drift }}= & \frac{4 e^{2}}{\rho \theta_{B}+2 x}\left(\lambda\left(z-z_{D}\right)-\lambda\left(z-z_{C}\right)\right) \\
& +\int_{z-z_{D}}^{z} \frac{4 e^{2}}{\rho \phi+2 x} \frac{\partial \lambda\left(z^{\prime}\right)}{\partial z^{\prime}} d z^{\prime},
\end{aligned}
$$

where $\rho$ is the bending radius, $\theta_{B}$ is the total bend angle, $x$ is the subsequent drift coordinate, $\phi$ is the angular displacement in the bend, $\lambda$ is the normalized longitudinal distribution of the bunch, $z$ and $z^{\prime}$ are the internal bunch coordinates of the observing and source electron, respectively, and $z_{A}, z_{B}, z_{C}$, and $z_{D}$ are the slippage conditions. In the ultrarelativistic regime, the slippage conditions reduce to [13]

$$
\begin{aligned}
& z_{A}=\frac{\rho \phi^{3}}{6}, \\
& z_{B}=\frac{\rho \phi^{3}}{24}, \\
& z_{C}=\frac{\phi^{2}}{6}(\rho \phi+3 x), \\
& z_{D}=\frac{\rho \phi^{3}}{24}\left(\frac{\rho \phi+4 x}{\rho \phi+x}\right) .
\end{aligned}
$$

The integration-by-parts implementation followed by Ref. [12] results in the boundary terms on the right of Eqs. (1) and (2) and the integration over the derivative of the longitudinal bunch distributions. The boundary terms are referred to as the "transient" CSR wakes and arise from the space charge field of the beam. In the steady-state regime, the transients tend to zero and the lower bound of the integral terms spans the entire bunch tail's domain i.e., the energy transfer, $d E$, is constant along the trajectory, $d s$.

The normalized rms energy spread is an important parameter that determines the beam's dynamics. It allows us to see the CSR's impact on the beam's energy spread and projected emittance. The induced CSR rms energy spread for an electron bunch traversing a bending magnet in the steady-state regime $\left(z_{B} \rightarrow \infty\right)$ was first calculated for a
Gaussian longitudinal distribution by Y.S. Derbenev et al. in 1995 [10],

$$
\sigma_{\mathrm{CSR}}=0.246 \frac{N r_{e} L_{B}}{\gamma \rho^{2 / 3} \sigma_{z}^{4 / 3}},
$$

where $N$ is the electron population, $r_{e}$ is the classical electron radius, $L_{B}$ is the bend length, $\gamma$ is the beam energy normalized by the electron's rest mass, and $\sigma_{z}$ is the bunch length.

Deriving accessible rms expressions in place of the CSR integrals is of great utility. Unfortunately, due to the mathematical complexity, no such calculation, to date, has been published for a beam traversing a bend in the transient regime or in the subsequent drift section.

CSR is inherently a three-dimensional problem in space but there is a limit to which the dynamics will depend on the transverse dimensions,

$$
\sigma_{x, y} \gg \sigma_{z}\left(\frac{\rho}{\sigma_{z}}\right)^{1 / 3},
$$

where $\sigma_{x, y}$ is the spot size of the beam in either transverse dimension. This limit is referred to as the Derbernev criterion [10]. Though this limit is typically satisfied throughout the accelerator, it can be violated in the middle of a 4-Bend bunch compressor chicane where the beam's spot size is large. Though theory would suggest that a model beyond one dimension would be necessary in these instances, studies have shown that the disagreement with a higher dimensional implementation is small. In this study, we will be working strictly with the 1D CSR formalism.

\section{CSR rms EXPRESSIONS}

In this section, we develop expressions for the CSR rms energy spread induced on a beam in a bend transitioning into steady state with and without compression and the subsequent drift in lieu of the integral expressions. All derivations take advantage of approximations that can be assumed for various XFEL applications.

\section{A. CSR energy spread of a beam transitioning to steady state}

The $\sigma_{\mathrm{CSR}}$ expression [Eq. (4)] was derived in the steadystate regime. But, a beam must first transition from the transient regime, and in some cases, steady state is never achieved. The steady-state rms expression is inadequate in describing this transitional state and tends to overestimate the induced rms energy spread. This is attributed to it including all of the tail electrons in the convolution integral, whereas the extent should be limited by the slippage condition [Eq. (3)]. We would like to have a more

\footnotetext{
${ }^{1}$ The paper cited has an incorrect leading coefficient of 0.22 .
} 
comprehensive expression to describe the rms energy spread of this transitional state.

To start, let us outline a set of assumptions for the CSR dynamics in the transient regime. First, we recognize that a key feature of the steady-state regime lies in the integration limits of Eq. (1), i.e., the amount of trailing electrons contributing to the wakefield. In the standard definition, steady state is achieved once the integration bounds encompass the entire tail-end of the bunch for any observing position, $z$, within the bunch, or the slippage condition exceeds $\sigma_{z}$. However, this requirement for the slippage to surpass $\sigma_{z}$ is arbitrary and the slippage required to encompass the entire tail-end of the beam will vary for different longitudinal positions; the tail-end of the beam would require a shorter slippage to reach steady-state and vice versa for the head of the beam. To account for this, we include an empirical parameter, $P_{\mathrm{SS}}$, on the steady-state condition,

$$
z-z^{\prime}=\sigma_{z}=P_{\mathrm{SS}} \frac{\rho \phi^{3}}{24}
$$

The $P_{\mathrm{Ss}}$ parameter is intended to roughly correct the slippage condition to describe the entire beam's transition to the steady-state regime (Fig. 1, blue regions). $P_{\mathrm{SS}}$ is calculated with a least-square optimization of our data sets (Sec. III D) and is found to be roughly 1.6.

Second, in the transient regime, the two boundary terms in Eq. (1) will be dominant. At the entrance of the bend $(\phi \sim 0)$, the slippage will be negligible and the two wakes will have near-perfect cancellation. At small angles $\left(\phi<\left(\frac{6 P_{\mathrm{SS}} \sigma_{z}}{\rho}\right)^{1 / 3}\right)$, the wakes will begin to separate due to the difference in their slippage rates and the induced CSR rms energy spread will begin to increase. At moderate angles $\left(\left(\frac{6 P_{\mathrm{SS}} \sigma_{z}}{\rho}\right)^{1 / 3}<\phi<\left(\frac{24 P_{\mathrm{SS}} \sigma_{z}}{\rho}\right)^{1 / 3}\right)$, the preceding drift transient $\left(\propto z_{A}\right)$ will have slipped past the head of the beam
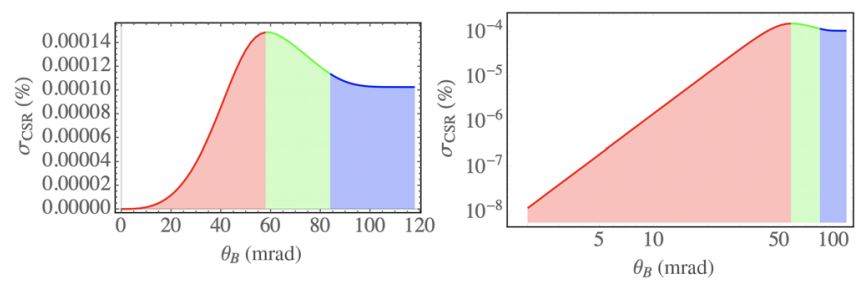

FIG. 1. The CSR rms induced energy spread using numerical integration from the two transient terms in Eq. (1) for various bend angles of a $100 \mathrm{pC}, 1.8 \mathrm{GeV}$ electron beam $\left(\sigma_{z}=100 \mu \mathrm{m}\right)$ traversing a bending magnet $(\rho=10 \mathrm{~m})$. The plots are divided into three regions. Red region: The small angle region where both wakes are present and beginning to separate. Green region: The preceding drift transient, $\propto z_{A}$, has slipped past the beam and only the bend transient, $\propto z_{B}$, is present. Blue region: The bend transient slips past the beam and the CSR interaction is in steady state. The right plot is a Log-Log plot of the energy CSR rms energy spread and shows the different slopes of the three regimes. and no longer sustain energy transfer. Meanwhile, the bend transient $\left(\propto z_{B}\right)$ will continue to slip toward the head of the beam and due to its opposite sign, undo some of the energy transfer from the entrance drift transient i.e., the energy spread will slightly decrease. Finally, for large angles $\left(\phi>\left(\frac{24 P_{\mathrm{Ss}} \sigma_{z}}{\rho}\right)^{1 / 3}\right)$, the transient wakes will have slipped in front the head of the bunch and no energy transfer will continue. At this point, steady state will be achieved and the energy spread induced by the transients will remain constant. This process is outlined in Fig. 1.

To begin our derivation, we separate the transient and steady-state regimes by the angle of transition, $\theta_{T}$, which can be solved from Eq. (6),

$$
z-z^{\prime}=\sigma_{z}\left(\theta_{T}\right)=P_{\mathrm{SS}} \frac{\rho \theta_{T}^{3}}{24},
$$

where $\sigma_{z}\left(\theta_{T}\right)$ is the bunch length as a function of angle through the bend and $P_{\mathrm{SS}}$ is the steady-state parameter. The transient regime is dominated by the slipping wakes so we ignore the integral portion of Eq. (1),

$$
\begin{aligned}
\left.\frac{d E}{d s}\right|_{\text {Transient }} & =\frac{4 e^{2}}{\rho \phi}\left(\lambda\left(z-z_{B}\right)-\lambda\left(z-z_{A}\right)\right) \\
& =\frac{4 e^{2}}{\rho \phi}\left(\lambda_{i}\left(z-z_{B}\right)-\lambda_{i}\left(z-4 z_{B}\right)\right),
\end{aligned}
$$

where $\lambda_{i}$ is the initial bunch distribution at the entrance of the bend. Calculating the rms of this expression cannot be done analytically, so we will need to apply the following simplifications. First, since we are in the transient regime, we can assume the angular displacement within the bend is small, $\phi<\theta_{T}$, and so an expansion of the wakes to the second order in $\phi$ is allowed,

$$
\left.\frac{d E}{d s}\right|_{\text {Transient }} \approx-z \frac{e^{2} \phi^{2}}{2 \sigma_{z i}^{2}} \lambda_{i}(z),
$$

where $\sigma_{z i}$ is the rms bunch length at the entrance of the bend. We integrate this expression through the bend to get the total energy change,

$$
\begin{aligned}
E_{\text {Transient }} & =\int_{0}^{\theta_{T}}-z \frac{e^{2} \phi^{2}}{2 \sigma_{z i}^{2}} \lambda_{i}(z) \rho d \phi \\
& =-z \frac{e^{2} \rho \theta_{T}^{3}}{6 \sigma_{z i}^{2}} \lambda_{i}(z),
\end{aligned}
$$

and now, calculate the rms,

$$
\sigma_{\text {Transient }}=0.029 \frac{N r_{e} L_{B} \theta_{T}^{2}}{\gamma \sigma_{z i}^{2}} .
$$

The expression above will account for the CSR-induced rms energy spread while the beam remains in the transient 
regime. If beam transitions into steady state, Eq. (4) can be used to find the CSR rms energy spread for the residual portion of the bend $\left(\phi>\theta_{T}\right)$. The total CSR rms energy spread is simply the sum of the transient and steady-state expressions and the results can be organized conveniently,

$$
\sigma_{\mathrm{Bend}}= \begin{cases}0.029 \frac{N r_{e} L_{B} \theta_{T}^{2}}{\gamma \sigma_{z i}^{2}}, & \text { if } \theta_{T}>\theta_{B} \\ \frac{N r_{e}}{\gamma}\left(0.029 \frac{L_{B} \theta_{T}^{2}}{\sigma_{z i}^{2}}+0.246 \frac{\rho^{1 / 3}\left(\theta_{B}-\theta_{T}\right)}{\sigma_{z i}^{4 / 3}}\right), & \text { if } \theta_{T}<\theta_{B} .\end{cases}
$$

\section{B. CSR energy spread of a compressing beam transitioning to steady state}

In XFELs, the electron beam must be compressed to have a suitable peak current for lasing. The compression is typically done with the use of magnetic bunch compressor chicanes (Fig. 9). The longitudinal movement of particles in the chicane is quantified by the $R_{56}$,

$$
R_{56}=\int \frac{\eta(s)}{\rho} d s,
$$

where the integral above is integrated over the bending magnets of the chicane. The $R_{56}$ relates the first-order shift of longitudinal position to a particle's normalized energy deviation, $\Delta z=R_{56}(s) \delta_{E}$, which can be further related to the change of the rms bunch length of a beam, $\sigma_{z}(s)=\left(1+R_{56}(s) h\right) \sigma_{z i}$, where $h$ is the first-order energy chirp of the beam. For our purposes, we assume a simple linearized model of the compression in the bends which is valid when $\Delta \sigma_{z}<\sigma_{z}$,

$$
\sigma_{z}(\phi)=\sigma_{z i}+\left(\frac{\Delta \sigma_{z}}{\theta_{B}}\right) \phi,
$$

where $\Delta \sigma_{z}=\sigma_{z f}-\sigma_{z i}, \sigma_{z i}$ is the bunch length at the entrance of the bend, $\sigma_{z f}$ is the bunch length at the exit of the bend, and $\theta_{B}$ is the total bend angle.

Often, a beam will be compressed into steady state while traversing a bend and the compression will serve as a catalyst to steady state. Instead of Eq. (4), an amended CSR rms expression taking into account compression could be

$$
\frac{d \sigma_{\mathrm{CSR}}(\phi)}{\rho d \phi}=0.246 \frac{N r_{e}}{\gamma \rho^{2 / 3}\left(\sigma_{z i}+\left(\frac{\Delta \sigma_{z}}{\theta_{B}}\right) \phi\right)^{4 / 3}} .
$$

This expression tends to overestimate the energy modulation as it ignores the initial portion of the bend where the beam is dominated by the transient regime. In what follows, we will derive an expression that connects the transient and steady-state portions of the bend along with the effects of compression.

To start, we will assume that the compression of the bunch $(\propto \phi)$ is negligible relative to the slippage of the wake $\left(\propto \phi^{3}\right)$ while in the transient regime. Hence, we can incorporate the effects of compression into the transient terms with the use of a compression parameter, $A_{\mathrm{Comp}}=$ $1+\frac{\left(\sigma_{z i}-\sigma_{z f}\right)}{\sigma_{z i}}=\left(2 \sigma_{z i}-\sigma_{z f}\right) / \sigma_{z i}$, on $\theta_{T}$ in Eq. (12) that linearly increases as the bunch is being compressed and tends to unity when there is no compression. This will describe the transient portion of the bend. We use the angle of transition, $\theta_{T}$, given in Eq. (7) to separate the transient $\left(\phi<\theta_{T}\right)$ and steady-state $\left(\phi>\theta_{T}\right)$ regimes. So, before the beam transitions into steady-state, the transient regime will account for the energy modulation up to the angle of transition $\left(\phi=\theta_{T}\right)$. After the beam transitions to steady state, we can use the amended CSR rms expression [Eq. (16)] integrated through the remaining portion of the bend $\left(\theta_{T}<\phi<\theta_{B}\right)$. The total energy spread will just be a sum of the transient and steady-state regimes,

$$
\begin{aligned}
\sigma_{\text {Comp }}= & 0.029 \frac{N r_{e} L_{B}\left(A_{\mathrm{Comp}} \theta_{T}\right)^{2}}{\gamma \sigma_{z i}^{2}} \\
& +\int_{\theta_{T}}^{\theta_{B}}\left(0.246 \frac{N r_{e}}{\gamma \rho^{2 / 3}\left(\sigma_{z i}+\left(\frac{\Delta \sigma_{z}}{\theta_{B}}\right) \phi\right)^{4 / 3}}\right) \rho d \phi,
\end{aligned}
$$

where $\sigma_{z i}$ and $\sigma_{z f}$ are the initial and final bunch lengths, respectively. The results can be organized as follows:

$$
\sigma_{\text {Comp }}= \begin{cases}0.029 \frac{N r_{e} L_{B}\left(2 \sigma_{z i}-\sigma_{z f}\right)^{2} \theta_{T}^{2}}{\gamma \sigma_{z i}^{4}}, & \text { if } \theta_{T}>\theta_{B} \\ \frac{N r_{e} L_{B}}{\gamma}\left(0.029 \frac{\left(2 \sigma_{z i}-\sigma_{z f}\right)^{2} \theta_{T}^{2}}{\sigma_{z i}^{4}}+\frac{0.738}{\rho^{2 / 3}\left(\sigma_{z i}-\sigma_{z f}\right)}\left(\frac{1}{\sigma_{z f}^{1 / 3}}-\frac{1}{\left(\sigma_{z i}-\left(\frac{\sigma_{z i}-\sigma_{z f}}{\theta_{B}}\right) \theta_{T}\right)^{1 / 3}}\right)\right), & \text { if } \theta_{T}<\theta_{B} .\end{cases}
$$

\section{CSR energy spread of a beam traversing a subsequent drift section}

The CSR in the subsequent drift section of a bending magnet can generate significant energy modulation and, in some instances, be greater than that of a bend. The difficulty in solving the drift CSR integral [Eq. (2)] is in it requiring numerical 
methods even for simple bunch distributions e.g., a uniform or Gaussian distribution. Again, a simple expression to describe the CSR-induced rms energy spread in a drift is desired. To start, we separate the CSR dynamics into a transient and steady-state regime and discuss their characteristics.

The transient regime is described by the combined effect of the boundary terms and integral term of Eq. (2). As an observing electron exits the bend and travels along the subsequent drift, the transient wakes will begin to slip in front of the bunch at different rates [Eq. (3)]. The $z_{C}$ slippage factor is linearly dependent on $x$ and causes the wake to slip in front of the bunch quickly and is only active for small drift lengths, $3 x \ll L_{B}$ (Fig. 2, left). Whereas the $z_{D}$ slippage factor depends weakly on $x$ and causes the wake to slip to a localized position of $z=\frac{\rho \phi^{3}}{6}$ within the bunch for $x \gg L_{B}$ (Fig. 2, right). Meaning, for short bends $\left(z_{D}\left(\theta_{B}\right)<\sigma_{z}\right)$, the transient wake with the $z_{D}$ dependence will provide sustained energy modulation along all drift positions until its $1 / x$ dependence suppresses it. Additionally, the transient regime will include a contribution from the integral term for short bend angles/lengths $\left(z_{D}\left(L_{B}, \theta_{B}\right) \ll \sigma_{z}\right)$. An observing electron will only be interacting with a segment of the trailing beam, determined by the slippage, at any given point. An increase of the bend geometry such that, $z_{D}\left(L_{B}, \theta_{B}\right) \gg \sigma_{z}$, will transition the integral term to steady state.

The steady-state regime is described by the integral term integrated over the full extent of the beam. Here, the $z_{C}$ transient wake has slipped out of range of the electron bunch and the $z_{D}$ transient wake has slipped to a static position of $z=\frac{\rho \phi^{3}}{6}$ which, depending on the bunch length, may have slipped out of range. Unlike the bend CSR steady-state regime [Eq. (4)], the drift steady-state's energy transfer is attenuated by the $1 / x$ dependence; at some $x$ location, the wake will be fully suppressed and energy transfer will stop.

We start the mathematical implementation by deriving an expression for only the $z_{D}$ transient wake. The dominant behavior of this wake is in its decay over the drift distance. Therefore, we can approximate it by completely ignoring the slippage over the bunch distribution,
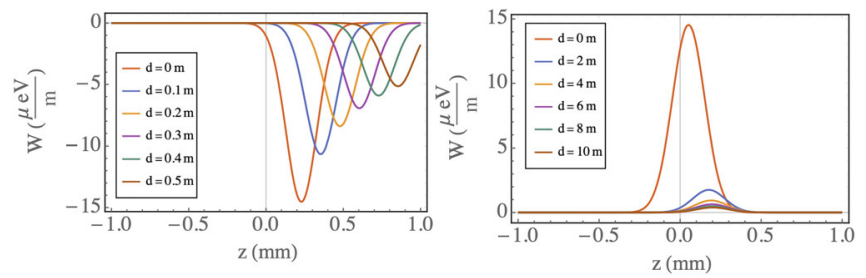

FIG. 2. The drift space CSR's boundary terms [Eq. (2)] energy modulation along the bunch's internal coordinate, $z$, for various positions along the drift. Left: The transient wake containing the $z_{C}$ slippage term. Right: The transient wake containing the $z_{D}$ slippage term.

$$
\left.\frac{d E}{d s}\right|_{\text {Drift }} \approx \frac{4 e^{2}}{\rho \theta_{B}+2 x} \lambda\left(z-z_{D}\right) \approx \frac{4 e^{2}}{\rho \theta_{B}+2 x} \lambda(z) .
$$

In this approximated form, the transient can be integrated over the drift distance, $d$, to get the total energy modulation,

$$
E_{\text {Total }}=\int_{0}^{d} \frac{4 e^{2}}{\rho \theta_{B}+2 x} \lambda(z) d x
$$

and the rms energy spread induced by the transient is straight forward to calculate,

$$
\sigma_{\text {Drift }}=\sqrt{\frac{2 \sqrt{3}-3}{3 \pi}} \frac{N r_{e}}{\gamma \sigma_{z}} \log \left(\frac{2 d}{L_{B}}+1\right) .
$$

The unity inside the log's argument ensures the rms is positive and the $\frac{2 d}{L_{B}}$ term controls the rms's evolution along the drift, a feature we will utilize later when we introduce the steady-state regime into the expression.

Next, the $z_{C}$ transient wake needs to be included, but because of its $x$-dependence this is not simple, or even possible, to do analytically. To incorporate it, we will use simple limiting cases between the wakes dynamics. First, we will use the fact that the two transient terms are of opposite sign and at the beginning of the drift, they have the same magnitude and cancel each other, $\sigma_{\text {Drift }}(d=0) \sim 0$. Along the drift, the wakes begin to separate at a rate $\propto \rho \phi^{3}$ due to their difference of slippage factors, $z_{D}-z_{C}$. Lastly, the $z_{C}$ wake will slip out of range due to its strong dependence on $x$ and we will be left with only the $z_{D}$ wake $\left[\sigma_{\text {Drift }}\right.$ from Eq. (21)]. To model this dynamic, we introduce an exponential factor in front of Eq. (21),

$$
\begin{aligned}
& \sigma_{\text {Drift }}=\left(1-\exp \left(-\xi\left(d, \sigma_{z}\right) \rho \theta_{B}^{3}\right)\right) \\
& \times\left(\sqrt{\frac{2 \sqrt{3}-3}{3 \pi}} \frac{N r_{e}}{\gamma \sigma_{z}} \log \left(\frac{2 d}{L_{B}}+1\right)\right) .
\end{aligned}
$$

The exponential factor should model the $z_{C}$ transient wake's short interaction window with the beam. We chose a factor that is zero at $d=0$ and is normalized to unity for $d \gg 1$ with an overall effect similar to the logistic/sigmoid function of Fermi-Dirac statistics. The argument of the exponential factor, $\xi\left(d, \sigma_{z}\right)$, should be an empirical function of the transient wake parameters is given by

$$
\xi\left(d, \sigma_{z}\right)=\xi_{o} d^{1 / 2} \sigma_{z}^{-3 / 2},
$$

where $\xi_{o}$ is a unitless constant equal to 0.132 calculated with a least-square optimization of our simulation data (see Sec. III D). The $\xi$ function contains the scaling of the transient parameters and will represent the evolution of the $z_{D}$ and $z_{C}$ wakes extrapolated from simulation parameter scans. The choice of the $\rho \theta_{B}^{3}$ term in the exponential was 
made to emulate the form of the difference of slippage rates [Eq. (3)] between the transients. The factors of $d^{1 / 2}$ and $\sigma_{z}^{-3 / 2}$ are empirically chosen to best fit the data scans and to provide a unitless exponential function.

Now, to incorporate the steady-state regime, we first recognize that the wake in the drift section (along $x$ ) after a bend is essentially the CSR wakefield from the bend copropagating with the beam. If we assume that the beam has reached steady state in the bend before entering the drift then we can equivalently allow the integration's lower bound [Eq. (2)] to negative infinity. Second, as the CSR wake propagates into the drift, its power will decay as $x^{-1}$ as radiating electrons leave the bend. Integrating over the drift distance, $x$, the energy transfer will tend as the natural $\log$ and contain a singularity at $x=0$ which we circumvent by restricting the integration limits over drift intervals where the entire bunch has transitioned out of the bend $\left(x \geq \sigma_{z}\right)$.

To begin implementation, we calculate the drift CSR integral using a different approach than previously published [13]. The standard derivation is obtained via integration-by-parts. Instead, we opt to integrate the twoelectron kernel with the longitudinal bunch distribution,

$$
\left.\frac{d E}{d s}\right|_{\text {Drift }}=\int_{z-z_{D}}^{z} \frac{-32 e^{2}(\rho \phi+x)^{2}}{\phi^{2}(\rho \phi+2 x)^{4}} \lambda\left(z^{\prime}\right) d z^{\prime},
$$

where the two-electron wake is taken from Stupakov and Emma in the ultrarelativistic regime [13]. We can change the integration variable using the Jacobian of transformation, $d z^{\prime}=\frac{-\rho \phi^{2}}{8}\left(\frac{(\rho \phi+2 x)}{(\rho \phi+x)}\right)^{2} d \phi$, and integrate over $\phi$,

$$
\left.\frac{d E}{d s}\right|_{\text {Drift }}=\int_{\theta_{B}}^{0} \frac{4 e^{2} \rho}{(\rho \phi+2 x)^{2}} \lambda\left(z-z_{D}\right) d \phi .
$$

The CSR integral in this form contains all of the information of Eq. (2) except for the $z_{C}$ boundary term. Next, we expand the bunch distribution for small $\phi$ to evaluate the integral,

$$
\begin{aligned}
\left.\frac{d E}{d s}\right|_{\text {Drift }} & =\int_{\theta_{B}}^{0} \frac{4 e^{2} \rho}{(\rho \phi+2 x)^{2}}\left(\frac{N}{\sqrt{2 \pi} \sigma_{z}} \exp \left(-\frac{z^{2}}{2 \sigma_{z}^{2}}\right)\right) d \phi \\
& \approx \frac{4 e^{2} \rho \theta_{B}}{2 x\left(\rho \theta_{B}+2 x\right)}\left(\frac{N}{\sqrt{2 \pi} \sigma_{z}} \exp \left(-\frac{z^{2}}{2 \sigma_{z}^{2}}\right)\right) \\
& \approx \frac{1}{\frac{2 x}{L_{B}}} \frac{4 e^{2} \lambda(z)}{\left(\rho \theta_{B}+2 x\right)},
\end{aligned}
$$

where we took only the zeroth-order term. The wake in its current form does not lead to an rms expression through any analytical means known to the authors. But note that the zeroth-order steady-state wake has a similar form as the transient wake, Eq. (19), except for an additional $\left(2 x / L_{B}\right)^{-1}$ modulation factor. Physically, this wake dissipates faster along the drift than the $z_{D}$ transient wake with a similar profile and slippage behavior. We, therefore, hypothesize that the steady-state rms expression will have the same form as Eq. (22) but with a different evolution of the $\log$ function i.e., the $\frac{2 x}{L_{B}}$ factor in the log will have a different form. This should permit us to include the effects of the steady-state wake into our rms expression via an empirical function as we did with the $z_{C}$ wake,

$$
\begin{aligned}
\sigma_{\text {Drift }}= & \left(1-\exp \left(\xi\left(d, \sigma_{z}\right) \rho \theta_{B}^{3}\right)\right) \sqrt{\frac{2 \sqrt{3}-3}{3 \pi}} \\
& \times \frac{N r_{e}}{\gamma \sigma_{z}} \log \left(\chi\left(\sigma_{z}, \rho, \theta_{B}\right) \frac{2 d}{L_{B}}+1\right)
\end{aligned}
$$

where $\chi\left(\sigma_{z}, \rho, \theta_{B}\right)$ is a function representing the impact of the zeroth-order steady-state wake. The $\chi$ function should model the impact of the steady-state wake (a beam in full steady state should enhance the log's argument) and have the correct parameter scaling in the steady-state regime that of which was extrapolated from simulation. A simple quantifier of the steady-state's strength could be how far the beam will be in steady state for the given bend geometry which can be measured by ratio of the full bend angle, $\theta_{B}$, to the angle that steady state is achieved, $\theta_{\mathrm{SS}}$, for the $z_{D}$ slippage factor (in the long drift limit, $x \gg L_{B}$ ),

$\chi\left(\sigma_{z}, \rho, \theta_{B}\right)=\left(\frac{\theta_{B}}{\theta_{\mathrm{SS}}}\right)=\left(\frac{\theta_{B}}{\left(\frac{6 \sigma_{z}}{\rho}\right)^{1 / 3}}\right)=\chi_{o}\left(\frac{\rho \theta_{B}^{3}}{\sigma_{z}}\right)^{1 / 3}$,

where $\chi_{o}$ is a unitless constant equal to 0.525 that was determined with a least-square optimization of the simulation data (see Sec. III D). The $\chi_{o}$ constant is similar to $P_{\mathrm{SS}}$ of Sec. III A as it corrects the variation of the $z_{D}$ slippage requirement from a single $\sigma_{z}$ for different longitudinal slices along the bunch.

\section{Simulation results}

Comprehensive parameter scans were conducted to test the robustness of our CSR expressions. ELEGANT was used to simulate the CSR effect (incoherent synchrotron radiation was not included) in the bends and drifts for a Gaussian, $1.8 \mathrm{GeV}, 100 \mathrm{pC}$ electron beam. In the following, we will present the results of our expressions compared with ELEGANT and discuss the limits of applicability for each. A summary of these limits is given in Table I.

Parameter scans for Eq. (13) are shown in Figs. 3-5. Though the expression shows overall good agreement, there are regions where the limits of validity are violated. The main approximation in Eq. (13) was made in the transient portion of the expression where we expanded the wakes for small angles up to the second order. This approximation retains information about the initial 
TABLE I. Limits in which our CSR rms expressions breakdown.

\begin{tabular}{lccc}
\hline \hline Expression & Limit 1 & Limit 2 & Limit 3 \\
\hline Eq. (13), $\theta_{T}<\theta_{B}$ & $\phi \ll 1, \sigma_{z} \gg 1$ & $L_{B} \ll 1$ & $\sigma_{z} \ll 1$ \\
Eq. (18) & $\phi \ll \theta_{T}$ & $\sigma_{z} \ll 1$ & \\
Eq. (27) & $x \phi^{2} \ll \sigma_{z}$ & $\rho \theta_{B}^{2} \ll \sigma_{z}$ & $\rho \theta_{B}^{2} \gg \sigma_{z}$ \\
\hline \hline
\end{tabular}

separation of the two wakes (red region in Fig. 1) but not after. The separation of the transient wakes is described by the difference in their slippage rates,
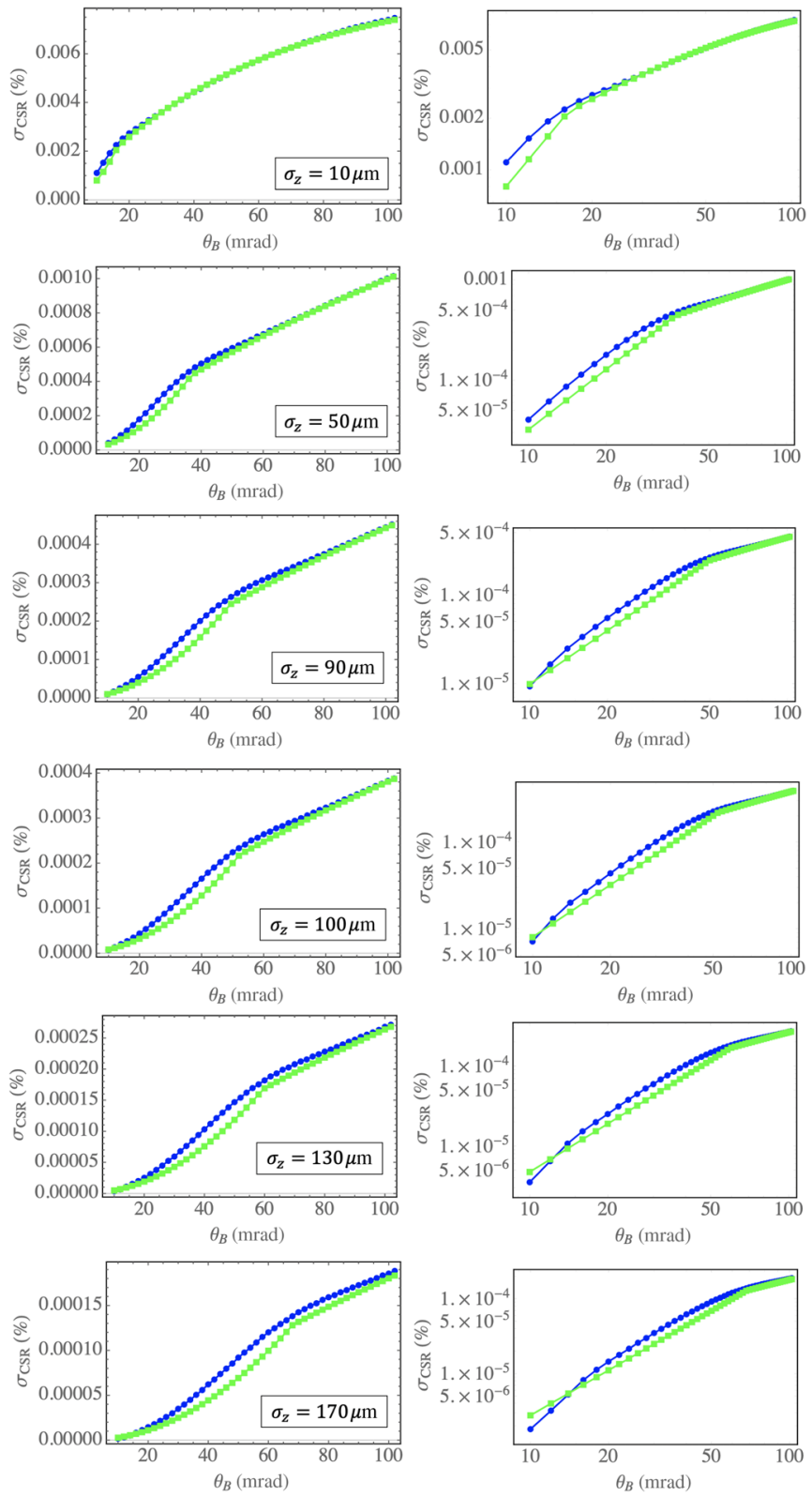

FIG. 3. The CSR rms energy spread versus bend angle of an electron beam traversing a bend $\left(L_{B}=0.55 \mathrm{~m}\right)$ for various initial bunch lengths. Left column: The CSR rms energy spread from ELEGANT (blue) and Eq. (13) (green). Right: Log-Log plot of the CSR rms energy spread.

$$
\Delta z_{\mathrm{SL}}=z_{A}-z_{B}=\frac{\rho \phi^{3}}{8}
$$

The lower limit of applicability occurs for $\Delta z_{S L} \ll \sigma_{z i}$, where the bunch length is long compared to wake separation. Here the separation is negligible compared to the overall bunch length and the transients effectively cancel each other rather than grow with a $\phi^{2}$ dependence [from Eq. (9)]. This limit breakdown is shown in Fig. 3 $(90-170 \mu \mathrm{m})$ where the angles are small, Fig. 4 $\left(\theta_{B}=0.01 \mathrm{rad}\right)$ for long bunch lengths, and Fig. 5 $(100-200 \mu \mathrm{m})$ when the bend length is at its shortest.

The upper limit of applicability occurs when the bunch length is short compared to the wake separation $\left(\Delta z_{S L} \gg \sigma_{z i}\right)$. Here, the short bunch length expedites the separation of the transients faster than $\frac{\rho \phi^{3}}{8}$ and the system transitions to the intermediate state (Fig. 1, green region). In this upper limit, steady state is achieved faster than with a $\phi^{2}$ dependence. This breakdown is shown in Fig. 4 $\left(\theta_{B}=0.07,0.10 \mathrm{rad}\right)$ for short bunch lengths.

The parameter scan for Eq. (18) is shown in Fig. 6. For this scan, we simulated a linearly chirped Gaussian bunch
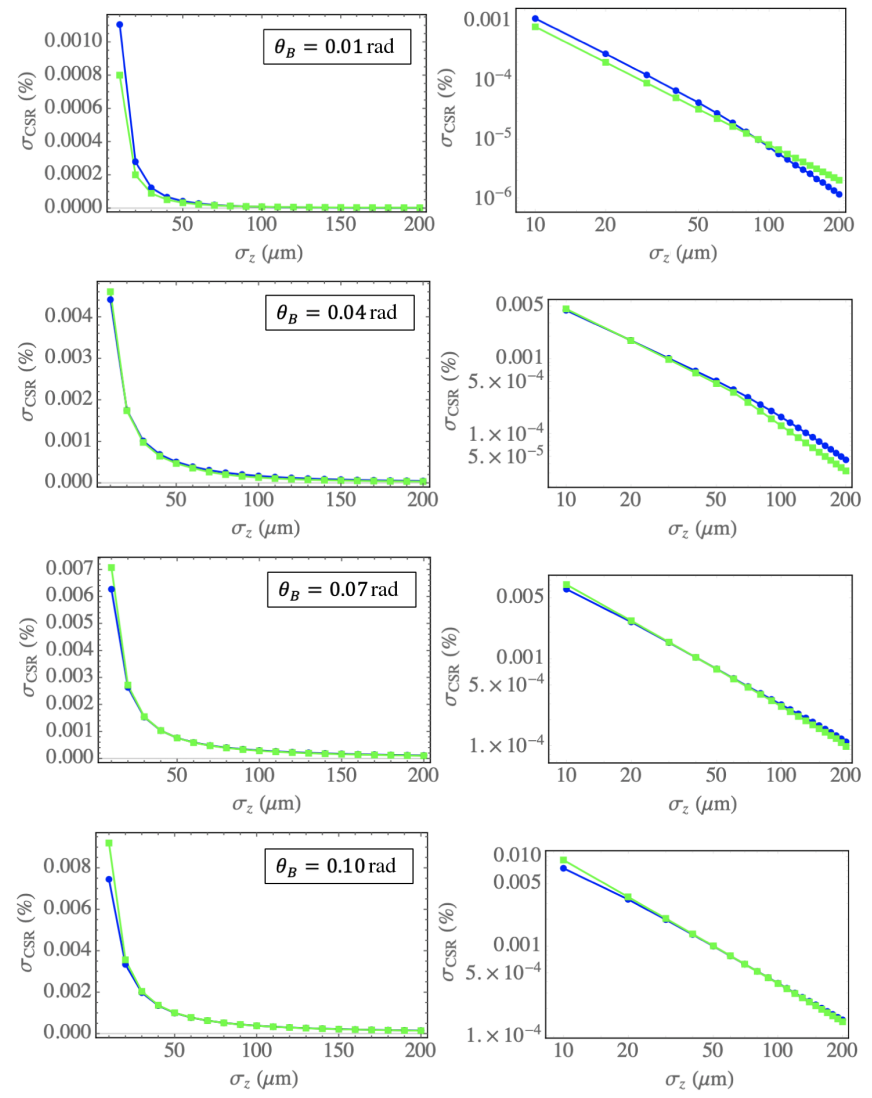

FIG. 4. The CSR rms energy spread versus initial bunch lengths of an electron beam traversing a bend $\left(L_{B}=0.55 \mathrm{~m}\right)$ for various bend angles. Left column: The CSR rms energy spread from ELEGANT (blue) and Eq. (13) (green). Right: Log-Log plot of the CSR rms energy spread. 

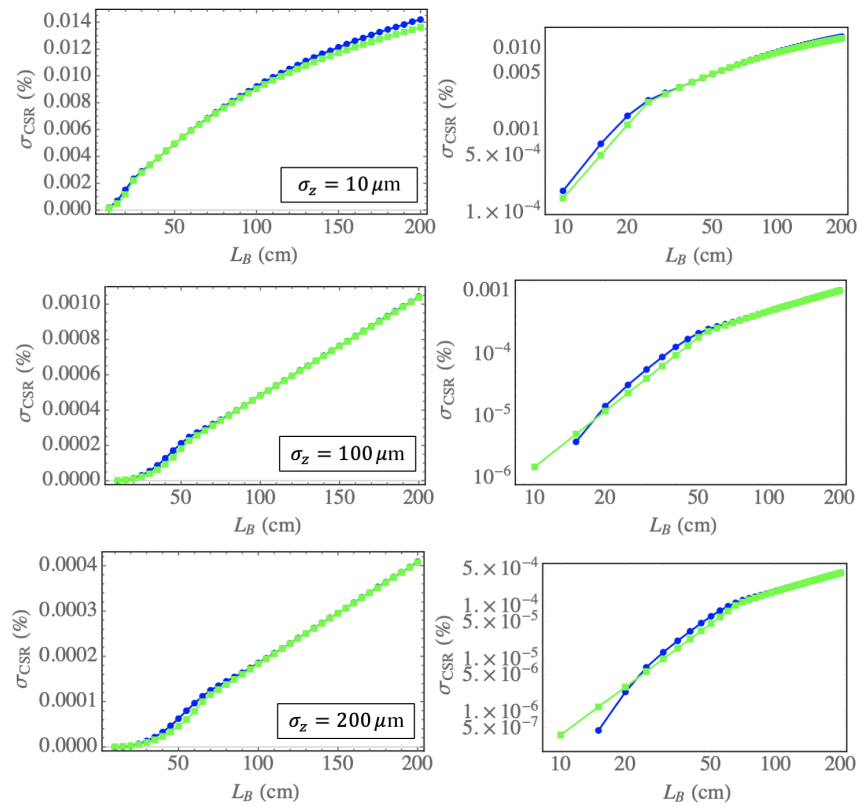

FIG. 5. The CSR rms energy spread for various initial bunch lengths traversing a bend of various bend lengths and constant bend curvature $(\rho=10 \mathrm{~m})$. Left column: The CSR rms energy spread from ELEGANT (blue) and Eq. (13) (green). Right: Log-Log plot of the CSR rms energy spread.

being compressed in the third bend of a 4-Bend chicane while scanning the bend angle. The angle is varied such that the beam will transition from the transient to the steadystate regime. The expression shows good agreement with ELEGANT, but the scaling at moderate angles is inaccurate. This is because our expression ignored the integral term while in the transient regime which underestimates the
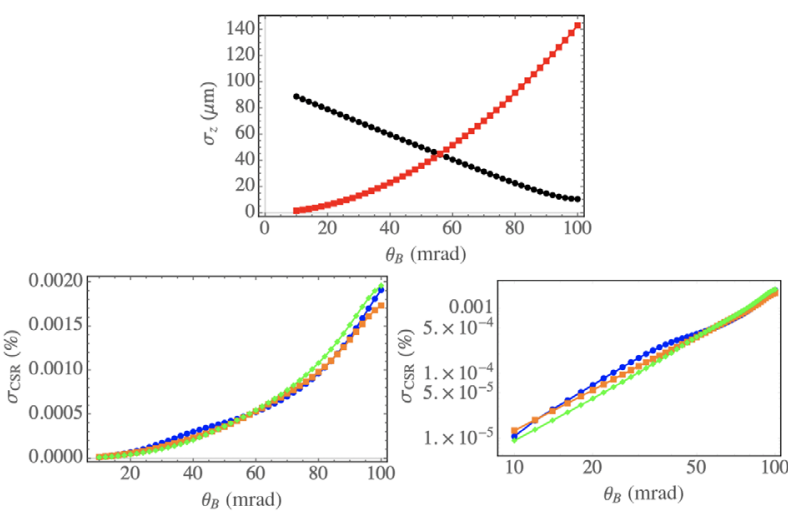

FIG. 6. The CSR rms energy spread of a chirped beam $\left(\sigma_{z i}=100 \mu \mathrm{m}\right)$ traversing the third bend $\left(L_{B}=0.55 \mathrm{~m}\right)$ of a 4-Bend chicane. The bend angle is varied and therefore the final bunch length out of the bend is as well. Top: The final bunch length (black line) and the modified slippage length (red line) from Eq. (7), the transition occurs at $\theta_{T}=0.056$ rad. Bottomleft: The CSR rms energy spread from ELEGANT (blue), numerical integration of Eqs. (1) and (2) (orange), and Eq. (18) (green). Bottom-right: Log-Log plot of the CSR rms energy spread. energy spread. Compression of the bunch length would intensify the CSR integral term [Eq. (1)] by expediting the slippage in the integration limits. This is also why at very small angles, where compression is negligible and the CSR interaction is dominated by the transient regime, we see agreement between our expression and ELEGANT.

The parameter scans for Eq. (27) are shown in Figs. 7 and 8. The drift CSR rms expressions have more strict limits of validity than the bend expressions. This is mainly due to the aggressive approximations made to reach an analytical result. First, we ignored the transient wake with the $z_{C}$ slippage factor when describing the transient regime [Eq. (19)] and we instead modeled it in the exponential factor which was purely empirical. So, the drift CSR rms expression's validity would be limited in regions where the $z_{C}$ transient wake is dominant: short drift distances, $x$, and short bend lengths, $L_{B}$, in Fig. 8, or small bend angles, $\theta_{B}$, in Fig. 7(c). These conditions will suppress the slippage factor and cause the wake to interact with the beam over a prolonged distance. Small bend geometries will also affect the steady-state description of our expression. For a bend geometry that's shorter than the bunch length, $\sigma_{z} \gg \frac{\rho \theta_{B}^{3}}{6}$, the $z_{D}$ wake will provide continuous energy transfer to the beam until its $1 / x$ dependence nullifies it which will
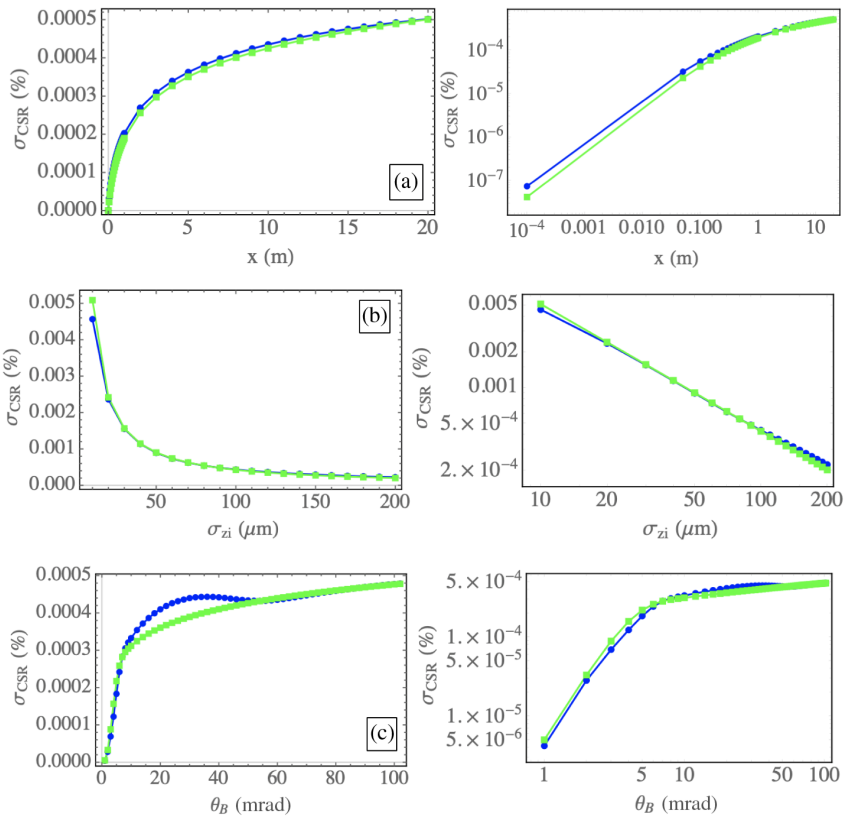

FIG. 7. The CSR rms energy spread (left column) and its LogLog (right column) plots of an electron beam traversing the subsequent drift space, $x$, of a bending magnet using ELEGANT (blue marker) and Eq. (27) (green marker): (a) Varying the drift space length for a $\sigma_{z i}=100 \mu \mathrm{m}$ beam exiting a $L_{B}=0.55 \mathrm{~m}$, $\theta_{B}=0.05 \mathrm{rad}$ bending magnet. (b) Varying the incoming bunch length, $\sigma_{z i}$, for a $L_{B}=0.55 \mathrm{~m}, \theta_{B}=0.05 \mathrm{rad}$ bending magnet and $x=10 \mathrm{~m}$ drift space. (c) Varying the bending angle, $\theta_{B}$, of a $L_{B}=0.55 \mathrm{~m}$ long bending magnet for a $\sigma_{z i}=100 \mu \mathrm{m}$ beam exiting into a $x=10 \mathrm{~m}$ drift space. 

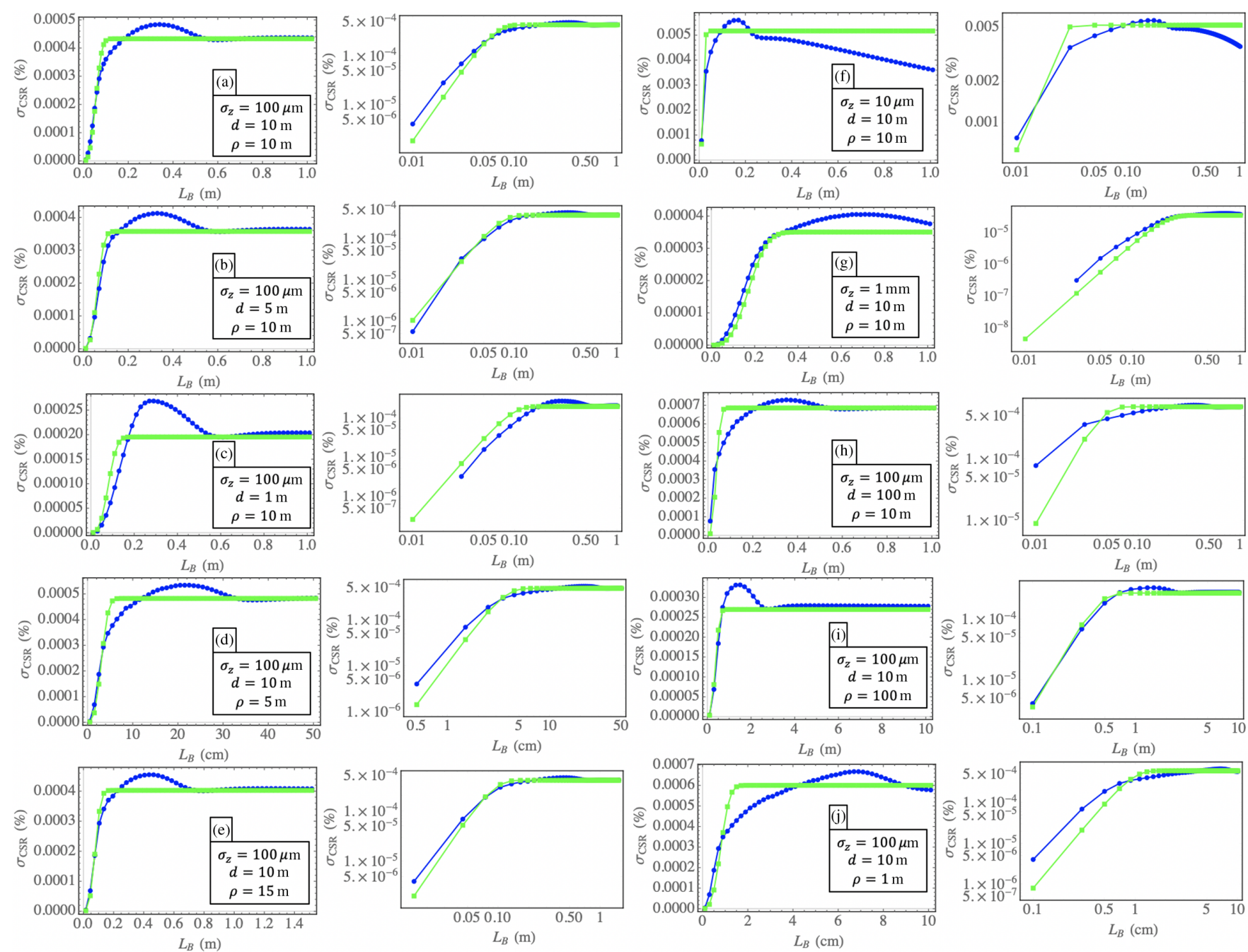

FIG. 8. The CSR rms energy spread (left column) and its Log-Log (right column) plots of an electron beam traversing the subsequent drift space of a bending magnet using ELEGANT (blue marker) and Eq. (27) (green marker) while varying the bending length, $L_{B}$, for various bend radii, drift lengths, and bunch lengths.

prolong the transition to steady state. This effect is evident in Fig. $8(\mathrm{~g})\left(\sigma_{z}=1 \mathrm{~mm}\right)$, where steady state is not reached through the entire $L_{B}$ scan. Conversely, for very short bunches, $\sigma_{z} \ll \frac{\rho \theta_{B}^{3}}{6}$, the zeroth-order approximation to the bunch distribution in Eq. (26) will no longer be accurate. The $z_{D}$ slippage factor will cause a large variation of energy modulation to the bunch distribution for relatively small bend angles and short drift distances.

\section{REAL BUNCH COMPRESSOR SYSTEMS}

Bunch compressor systems (Fig. 9) span a range of conditions that are ideal for testing our CSR expressions. In addition to finding the CSR rms energy spread, describing the emittance dilution at the end of the system is important. Using our expressions, we will calculate the rms energy spread and the bend-plane emittance growth due to CSR for various real bunch compressor systems and compare the results with ELEGANT.

\section{A. Emittance growth}

The geometric emittance at the end of a dispersive region under the influence of an energy modulation, $\delta_{E}$, is given by

$\epsilon_{f}=\sqrt{\epsilon_{o}^{2}+\epsilon_{o}\left(\beta_{x}\left\langle\Delta x^{\prime 2}\right\rangle+2 \alpha_{x}\left\langle\Delta x \Delta x^{\prime}\right\rangle+\gamma_{x}\left\langle\Delta x^{2}\right\rangle\right)+\Delta \epsilon^{2}}$,

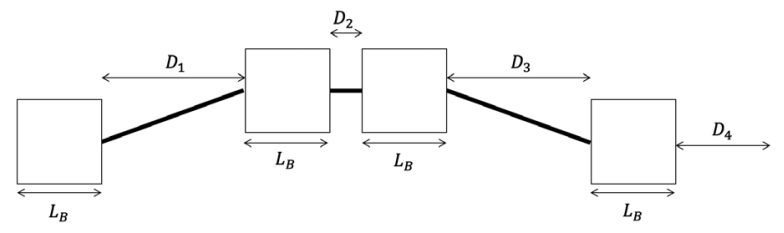

FIG. 9. Layout of 4-Bend bunch compressor (not to scale) used in the LCLS-II CuRF and LCLS-II-HE beam lines. 
TABLE II. LCLS-II CuRF and LCLS-II-HE BC1 and BC2 4-Bend chicane settings.

\begin{tabular}{lccccc}
\hline \hline Parameter & Symbol & Unit & LCLS-II CuRF (BC1, BC2) Values & LCLS-II-HE (BC1, BC2) Values \\
\hline Electron energy & $E_{o}$ & $\mathrm{GeV}$ & $(0.22,5.00)$ & $(0.25,1.80)$ \\
Net momentum compaction & $R_{56}$ & $\mathrm{~mm}$ & $(45.3,24.7)$ & $(54.6,37.0)$ \\
Floor length of each magnet & $L_{B}$ & $\mathrm{~m}$ & $(0.20,0.55)$ & $(0.20,0.55)$ \\
Floor length of first and third drift & $D_{1}, D_{3}$ & $\mathrm{~m}$ & $(2.45,9.87)$ & $(2.45,9.87)$ \\
Floor length of second drift & $D_{2}$ & $\mathrm{~m}$ & $(1.75,1.75)$ & $(1.75,1.75)$ \\
Floor length of fourth drift & $D_{4}$ & $\mathrm{~m}$ & $(10,10)$ & $(10,10)$ \\
Bend angle & $\left|\theta_{B}\right|$ & $\mathrm{mrad}$ & $(94,35)$ & $(103,42)$ \\
Dispersion peak & $\left|\eta_{\max }\right|$ & $\mathrm{mm}$ & $(250,362)$ & $(275,443)$ \\
\hline \hline
\end{tabular}

where $\epsilon_{o}$ is the beam's initial emittance, $\left(\beta_{x}, \alpha_{x}, \gamma_{x}\right)$ are the beam's Twiss parameters, $\left(\Delta x, \Delta x^{\prime}\right)$ are the coordinate and angular shifts due to the energy modulation, and $\Delta \epsilon$ is the phase space area occupied by the shifts. In the case of a chicane, if the energy modulation is coherent, the shifts add linearly and the second-order moments (see the Appendix) at the exit are

$$
\begin{aligned}
\left\langle\Delta x^{2}\right\rangle= & \left(\int\left(\eta(s)+\left(L_{T}-s\right) \eta^{\prime}(s)\right) \frac{\partial \sigma_{\delta_{E}}}{\partial s} d s\right)^{2}, \\
\left\langle\Delta x^{\prime 2}\right\rangle= & \left(\int \eta^{\prime}(s) \frac{\partial \sigma_{\delta_{E}}}{\partial s} d s\right)^{2}, \\
\left\langle\Delta x \Delta x^{\prime}\right\rangle= & \left(\int\left(\eta(s)+\left(L_{T}-s\right) \eta^{\prime}(s)\right) \frac{\partial \sigma_{\delta_{E}}}{\partial s} d s\right) \\
& \times\left(\int \eta^{\prime}(s) \frac{\partial \sigma_{\delta_{E}}}{\partial s} d s\right),
\end{aligned}
$$

where $\eta(s)$ and $\eta^{\prime}(s)$ are the dispersion and its derivative at the location of the energy modulation, $s, \sigma_{\delta_{E}}$ is the rms spread of the normalized energy modulation, and $L_{T}$ is the total length of the chicane. To use our expressions to calculate the emittance growth, we need to find the variation of the rms energy spread along the accelerator
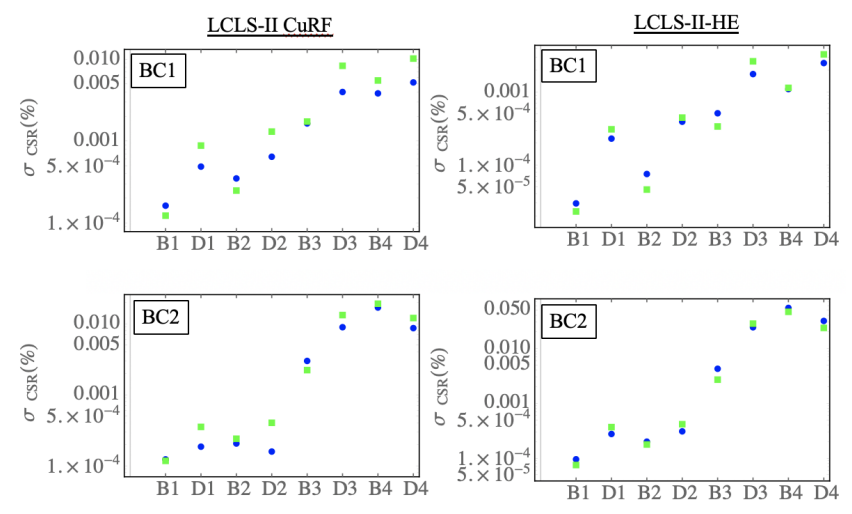

FIG. 10. The Log-Log plots of the CSR rms energy spread for each bend and drift element in the LCLS-II CuRF and LCLS-II first $(\mathrm{BC} 1)$ and second $(\mathrm{BC} 2)$ bunch compressors. The blue markers are obtained from ELEGANT simulations and the green markers our expressions. coordinate, $s$. This can be done by calculating the rms of the CSR wake without integrating for the total energy. Though, for a simpler implementation, one could linearize the total rms expressions [Eqs. (13), (18), and (27)]. Both methods show reasonable agreement with each other and the ELEGANT data.

In this section, we compare the results of our expressions for the 4-Bend bunch compressor chicanes of the LCLS-II CuRF and LCLS-II-HE (Table II). The two compressors input beams are generated from start-to-end simulations from the photoinjector through the linac. The bunch distribution is generated using ASTRA [21] and tracked through the linac to each compressor while simulating longitudinal space charge and CSR using ELEGANT.

\section{B. Simulation results}

A Log-Log plot of the results is plotted in Fig. 10. As expected, the expressions are less effective for $\mathrm{BC} 1$ than BC2 in both the LCLS-II CuRF and LCLS-II-HE beam lines. First, the relatively large bend angles of $\mathrm{BC} 1$ would affect the accuracy of the small angle expansion used in the expressions. Second, the parameters of $\mathrm{BC} 1$ in both beam lines give the largest difference between the wake separation factor, $\Delta z_{S L}$, in Eq. (29) and the initial bunch length, $\sigma_{z i}$. In particular, for LCLS-II CuRF's BC1, the discrepancy is largest for the first, second, and fourth bends, and for LCLS-II-HE's BC1, it's the largest for the first three bends. For both beam lines, the difference is smaller in $\mathrm{BC} 2$. Also, note the relatively large contribution from the drift sections in $\mathrm{BC} 1$ for both beam lines.

TABLE III. The LCLS-II CuRF and LCLS-II-HE BC1 and BC2 emittance calculation comparison between ELEGANT and our analytical expressions.

\begin{tabular}{lccc}
\hline \hline Location & Symbol & ELEGANT (\%) & $\begin{array}{c}\text { CSR } \\
\text { expressions }(\%)\end{array}$ \\
\hline LCLS-II CuRF BC1 & $\frac{\Delta \epsilon_{x}}{\epsilon_{o}}$ & 5 & 15 \\
LCLS-II CuRF BC2 & $\frac{\Delta \epsilon_{x}}{\epsilon_{o}}$ & 139 & 141 \\
LCLS-II-HE BC1 & $\frac{\Delta \epsilon_{x}}{\epsilon_{o}}$ & 1 & 2 \\
LCLS-II-HE BC2 & $\frac{\Delta \epsilon_{x}}{\epsilon_{o}}$ & 269 & 299 \\
\hline \hline
\end{tabular}


Finally, from the CSR rms energy spread, we can calculate the emittance growth in the bunch compressors. In Table III, we have listed the emittance growth of each bunch compressor for each beam line calculated by ELEGANT and using our newly developed expressions. The agreement is reasonable though our expressions tend to overestimate the emittance growth. This could be useful in tolerance studies where the maximum emittance dilution due to CSR is desired.

\section{CONCLUSION}

We have demonstrated, through several mathematical approximations, that the relative CSR rms energy spread for a beam transitioning into steady state and a beam propagating in a postbend drift section can be modeled. The expressions serve well for performing quick estimates of the CSR effect and its impact on the beam quality even in complex accelerator structures such as bunch compressor chicanes. The utility of such expressions can be used to highlight, and thereby circumvent, features of the CSR effect that may lead to potential degradation of the beam phase space quality.

\section{APPENDIX: EMITTANCE GROWTH DUE TO CSR IN DISPERSIVE REGIONS}

The infinitesimal angular change through a segment of accelerator is given by $d x^{\prime} \approx \frac{d s}{\rho_{o}}\left(1-\delta_{\mathrm{CSR}}\right)$. The total spatial and angular change due to CSR at a latter part of a system, $s_{f}$, can be found by transferring the kicks via the $R_{12}$ and $R_{22}$ matrix elements and integrating,

$$
\begin{aligned}
& x_{\mathrm{CSR}}\left(s_{f}\right)=-\int_{0}^{s_{f}} \frac{d s_{i}}{\rho_{o}} \delta_{\mathrm{CSR}}\left(s_{i}\right) R_{12}\left(s_{i}, s_{f}\right), \\
& x_{\mathrm{CSR}}^{\prime}\left(s_{f}\right)=-\int_{0}^{s_{f}} \frac{d s_{i}}{\rho_{o}} \delta_{\mathrm{CSR}}\left(s_{i}\right) R_{22}\left(s_{i}, s_{f}\right) .
\end{aligned}
$$

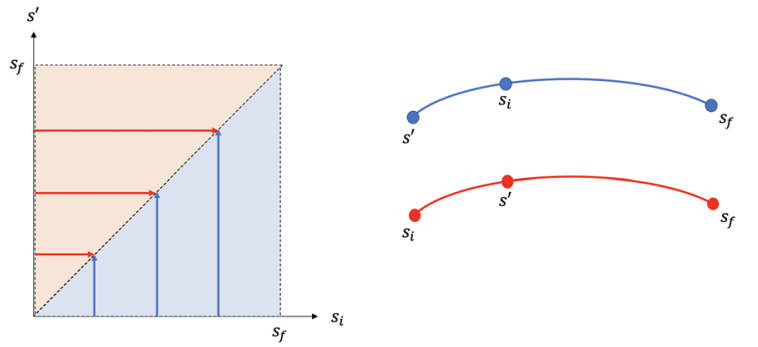

FIG. 11. Left: The change of the initial integration path (blue area) to the new path (red area). Right: The change of the variable orientation from the initial integration path (blue line) to the new path (red line).

The fractional energy change due to $\mathrm{CSR}, \delta_{\mathrm{CSR}}\left(s_{i}\right)$, is accumulative and can be integrated from past locations of the system,

$$
\begin{aligned}
x_{\mathrm{CSR}}\left(s_{f}\right) & =-\int_{0}^{s_{f}} \frac{d s_{i}}{\rho_{o}} R_{12}\left(s_{i}, s_{f}\right)\left(\int_{0}^{s_{i}} \frac{\partial \delta_{\mathrm{CSR}}\left(s^{\prime}\right)}{\partial s^{\prime}} d s^{\prime}\right) \\
& =-\int_{0}^{s^{\prime}} \frac{d s_{i}}{\rho_{o}} R_{12}\left(s_{i}, s_{f}\right)\left(\int_{0}^{s_{f}} \frac{\partial \delta_{\mathrm{CSR}}\left(s^{\prime}\right)}{\partial s^{\prime}} d s^{\prime}\right),
\end{aligned}
$$

$$
\begin{aligned}
x_{\mathrm{CSR}}^{\prime}\left(s_{f}\right) & =-\int_{0}^{s_{f}} \frac{d s_{i}}{\rho_{o}} R_{22}\left(s_{i}, s_{f}\right)\left(\int_{0}^{s_{i}} \frac{\partial \delta_{\mathrm{CSR}}\left(s^{\prime}\right)}{\partial s^{\prime}} d s^{\prime}\right) \\
& =-\int_{0}^{s^{\prime}} \frac{d s_{i}}{\rho_{o}} R_{22}\left(s_{i}, s_{f}\right)\left(\int_{0}^{s_{f}} \frac{\partial \delta_{\mathrm{CSR}}\left(s^{\prime}\right)}{\partial s^{\prime}} d s^{\prime}\right),
\end{aligned}
$$

where $s^{\prime}$ is a dummy index and the integration limits over the triangular region (Fig. 11) was rearranged. The matrix elements in the integrands can be decomposed using, $\mathbf{R}\left(s_{i}, s_{f}\right)=\mathbf{R}\left(s^{\prime}, s_{f}\right) \mathbf{R}\left(s_{i}, s^{\prime}\right)$, to give

$$
\begin{aligned}
x_{\mathrm{CSR}}\left(s_{f}\right) & =-\int_{0}^{s^{\prime}} \frac{d s_{i}}{\rho_{o}}\left(R_{11}\left(s^{\prime}, s_{f}\right) R_{12}\left(s_{i}, s^{\prime}\right)+R_{12}\left(s^{\prime}, s_{f}\right) R_{22}\left(s_{i}, s^{\prime}\right)\right) \int_{0}^{s_{f}} \frac{\partial \delta_{\mathrm{CSR}}\left(s^{\prime}\right)}{\partial s^{\prime}} d s^{\prime} \\
& =\int_{0}^{s_{f}}\left(R_{11}\left(s^{\prime}, s_{f}\right) \eta\left(s^{\prime}\right)+R_{12}\left(s^{\prime}, s_{f}\right) \eta^{\prime}\left(s^{\prime}\right)\right) \frac{\partial \delta_{\mathrm{CSR}}\left(s^{\prime}\right)}{\partial s^{\prime}} d s^{\prime}, \\
x_{\mathrm{CSR}}^{\prime}\left(s_{f}\right) & =-\int_{0}^{s^{\prime}} \frac{d s_{i}}{\rho_{o}}\left(R_{21}\left(s^{\prime}, s_{f}\right) R_{12}\left(s_{i}, s^{\prime}\right)+R_{22}\left(s^{\prime}, s_{f}\right) R_{22}\left(s_{i}, s^{\prime}\right)\right) \int_{0}^{s_{f}} \frac{\partial \delta_{\mathrm{CSR}}\left(s^{\prime}\right)}{\partial s^{\prime}} d s^{\prime} \\
& =\int_{0}^{s_{f}}\left(R_{21}\left(s^{\prime}, s_{f}\right) \eta\left(s^{\prime}\right)+R_{22}\left(s^{\prime}, s_{f}\right) \eta^{\prime}\left(s^{\prime}\right)\right) \frac{\partial \delta_{\mathrm{CSR}}\left(s^{\prime}\right)}{\partial s^{\prime}} d s^{\prime} .
\end{aligned}
$$

For a chicane bending system of length, $L_{T}$, our expression for the total spatial and angular shift at the end simplifies to

$$
x_{\mathrm{CSR}}\left(L_{T}\right)=\int_{0}^{L_{T}}\left(\eta\left(s^{\prime}\right)+\left(L_{T}-s^{\prime}\right) \eta^{\prime}\left(s^{\prime}\right)\right) \frac{\partial \delta_{\mathrm{CSR}}\left(s^{\prime}\right)}{\partial s^{\prime}} d s^{\prime},
$$




$$
x_{\mathrm{CSR}}^{\prime}\left(L_{T}\right)=\int_{0}^{L_{T}} \eta^{\prime}\left(s^{\prime}\right) \frac{\partial \delta_{\mathrm{CSR}}\left(s^{\prime}\right)}{\partial s^{\prime}} d s^{\prime}
$$

where the effects of focusing is ignored $\left(R_{21}=0\right)$, and the $R_{11}$ and $R_{22}$ matrix elements tend to unity.

[1] D. Douglas, Suppression and enhancement of CSR-driven emittance degradation in the IR-FEL driver, Thomas Jefferson National Accelerator Facility, Technical Report No. JLAB-TN-98-012, 1998.

[2] R. Hajima, A first-order matrix approach to the analysis of electron beam emittance growth caused by coherent synchrotron radiation, Jpn. J. Appl. Phys. 42, L974 (2003).

[3] F. Stulle, A. Adelmann, and M. Pedrozzi, Designing a bunch compressor chicane for a multi-TeV linear collider, Phys. Rev. ST Accel. Beams 10, 031001 (2007).

[4] Y. Jing, Y. Hao, and V. Litvinenko, Compensating effect of the coherent synchrotron radiation in bunch compressors, Phys. Rev. ST Accel. Beams 16, 060704 (2013).

[5] C. Mitchell, J. Qiang, and P. Emma, Longitudinal pulse shaping for the suppression of coherent synchrotron radiation-induced emittance growth, Phys. Rev. ST Accel. Beams 16, 060703 (2013).

[6] S. DiMitri, M. Cornacchia, and S. Spampinati, Cancellation of Coherent Synchrotron Radiation Kicks with Optics Balance, Phys. Rev. Lett. 110, 014801 (2013).

[7] S. DiMitri and M. Cornacchia, Merit functions for the linac optics design for colliders and light sources, Nucl. Instrum. Methods Phys. Res., Sect. A 735, 60 (2014).

[8] D.Z. Khan and T.O. Raubenheimer, LCLS-II bunch compressor study: 5-Bend Chicane, in Proceedings of the Conference FEL2014, Basel (JACoW, Basel, Switzerland, 2014).

[9] Y. Jiao, X. Cui, X. Huang, and G. Xu, Generic conditions for suppressing the coherent synchrotron radiation induced emittance growth in a two-dipole achromat, Phys. Rev. ST Accel. Beams 17, 060701 (2014).
[10] Y.S. Derbenev, E. L. Saldin, V.D. Shiltsev, and J. Rossbach, Microbunch radiative tail-head interaction, DESY, Technical Report No. DESY-TESLA-FEL-95-05, 1995.

[11] E. Saldin, E. Schneidmiller, and M. Yurkov, Radiative interaction of electrons in a bunch moving in an undulator, Nucl. Instrum. Methods Phys. Res., Sect. A 417, 158 (1998).

[12] E. Saldin, E. Schneidmiller, and M. Yurkov, On the coherent radiation of an electron bunch moving in an arc of a circle, Nucl. Instrum. Methods Phys. Res., Sect. A 398, 373 (1997).

[13] G. Stupakov and P. Emma, CSR wake for a short magnet in the ultrarelativistic limit, in Proceedings of the EPAC, Mulhouse, France (CERN, Geneva, Switzerland, 2002), p. 1479.

[14] C. Huang, T. Kwan, and B. Carlsten, Two dimensional model for coherent synchrotron radiation, Phys. Rev. ST Accel. Beams 16, 010701 (2013).

[15] A. Brynes, Understanding 1D to 3D coherent synchrotron radiation Effects, in Proceedings of the 39th Free Electron Laser Conference (FEL'19), Hamburg, Germany, 2019 (JACoW Publishing, Geneva, Switzerland, 2019), pp. 578-583.

[16] Y. Cai and Y. Ding, Three-dimensional effects of coherent synchrotron radiation by electrons in a bunch compressor, Phys. Rev. Accel. Beams 23, 014402 (2020).

[17] J. Tang and G. Stupakov (private communication).

[18] J. Arthur, P. Anfinrud, and P. Audebert, LCLS conceptual design report, SLAC Technical Report No. 7, 2002.

[19] T. O. Raubenheimer, The LCLS-II-HE, A high energy upgrade of the LCLS-II, in Proceedings of the 60th ICFA Advanced Beam Dynamics Workshop on Future Light Sources (JACoW, Shanghai, China, 2018), pp. 6-11.

[20] M. Borland, Elegant: A flexible SDDS-compliant code for accelerator simulation, Technical Report No. LS-287, Argonne National Lab., IL, USA, 2000.

[21] K. Flöttmann, Astra: A space charge tracking algorithm. User's manual available at https://www.desy.de/ mpyflo/, Astradokumentation (2013). 